\title{
ALVEOLAR RIDGE RECONSTRUCTION WITH TITANIUM MESH AND A MIXTURE OF AUTOGENOUS BONE GRAFT AND ALGIPORE
}

\author{
Hosam A. Afifi, Khaled M Elsayed, Bahaa E. Abd Rabboh
}

\begin{abstract}
Objective: The objective of the present study was to evaluate the efficiency of the alveolar ridge reconstruction with titanium mesh and a mixture of autogenous bone graft and bone substitutes (Algipore) in the atrophic mandible. Subjects and Methods: Clinical examination was made to all patients. Preoperative panoramic radiographs and cone beam CT were taken for every patient to determine alveolar bone height\& width. The change in the implant stability and bone density was measured using cone beam computerized tomography (CBCT) immediately, and after 3, 6 months of healing. All readings were recorded and analyzed statistically. Results: The mean horizontal bone gain was $3.4 \pm 2.0 \mathrm{~mm}$ \&the mean vertical bone gain was $5.4 \pm 3.4 \mathrm{~mm}$ after 6 months follow up, which was statistically significant._Conclusion: Excellent bone augmentation results both vertically and horizontally except for those of titanium mesh exposure.
\end{abstract}

Key Words: alveolar ridge reconstruction, titanium mesh, bone graft, algipore.

\section{INTRODUCTION}

Placement of osseo-integrated implants requires adequate height and width of alveolar bone as well as good bone quality, in many cases, the patients are presented with deficient alveolar bone either height or width that makes reconstruction obligatory ${ }^{(1-3)}$. Reconstruction of alveolar ridges for implant placement is still a challenging procedure, especially in the case of extensive vertical and horizontal bone atrophy. If the Implant stability or appropriate positioning cannot be achieved, alveolar ridge augmentation is needed. Several bone augmentation techniques have been introduced, of which autogenous bone grafting is the "gold standard" (4-5). Techniques to improve bone regeneration of segmental defects in the atrophic or dysmorphic (post-oncological) maxilla and mandible have been widely described since the 1990s.Distraction osteogenesis, splitting osteotomy, lateral ridge augmentation and guided bone regeneration are the most commonly applied methods ${ }^{(6-10)}$.
One of the main disadvantages of autogenous bone grafting is the lack of containment of the particulate graft, primarily due to the collapse of the soft tissue during function. During the postoperative period, compression or displacement of the graft has been described ${ }^{(11)}$.

The titanium mesh technique is one alternative, based on bone grafting with a stiff occlusive titanium membrane. Roccio and Colleagues have presented a surgical protocol for vertical ridge augmentation by using autogenous block bone grafts protected by a titanium mesh before implant placement ${ }^{(12-13)}$. Titanium meshes were introduced to limit or eliminate these problems and are used as a containment system for the particulate autogenous and alloplastic grafts. Studies performed by Louis et al. demonstrated that porous titanium meshes were a reliable containment system for the reconstruction of the maxilla and mandible, and that this material produced well-tolerated exposure, giving predictable results ${ }^{(14-15)}$.

\footnotetext{
* Dentist, Ministry of Health.

** Professor, department of Oral and Maxillofacial Surgery, Faculty of Dental Medicine, Boys, Cairo Al-Azhar University.

*** Assistant Professor, department of Oral and Maxillofacial Surgery, Faculty of Dental medicine, Boys, Cairo Al-Azhar University.
} 
Several benefits of the use of titanium mesh have been suggested. Titanium mesh provides superior space maintenance, a fundamental pre requisite for any bone regeneration procedure. Furthermore, the pores within the titanium mesh are thought to play a critical role in maintaining blood supply to a grafted defect ${ }^{(15)}$. Few reports in the literature address the clinical benefits obtained by using titanium mesh for localized alveolar ridge augmentation. In addition, limited knowledge is available regarding the resorption rate of the grafted area. The current study provides a clinical, radiographic, laboratory and histologic evaluation of the use of the mesh ${ }^{(16)}$.

\section{SUBJECTS AND METHODS}

In this study, 10 patients had alveolar ridge bone atrophy in the mandible. The patients were selected from Oral and Maxillofacial clinic of the Faculty of Oral and Dental Medicine, Al-Azhar University, Cairo, Boys. The patients were selected from the Out Patient Clinic of the Oral \& Maxillofacial Surgery Department, Faculty of Dentistry, AlAzhar University. The inclusion criteria of this study were; Patients of both sexes. Patients are free from any systemic diseases. Patients are in middle age between (20-35) years. Atrophic mandible Maximum vertical height above inferior alveolar canal must be $10 \mathrm{~mm}$ in the mandible.

The exclusion criteria were; Presence of any systemic diseases that affect osseointegration, Presence of occlusion discrepancies (cross bite and deep bite) and Para-functional habits (clenching and bruxsim), Presence of any periapical pathosis, Insufficient mouth opening to accommodate surgical instruments, Heavy smokers and alcohol abuse, Post head and neck radiation therapy and Pregnancy and Haematogical disease. Several clinical examinations were performed to exclude the presence of any disease or ulcer, any periodontal diseases and sufficient inter-arch space was inspected; the buccal and lingual mucosa of the mandibular alveolar ridge was palpated for the presence of any irregularity or tissue hyperplasia. Clinical records: Standardized preoperative photographs to the alveolar ridge were taken to the patients and Study casts were made to evaluate the condition of remaining dentition and ridges.

\section{Surgical procedure:}

1. The study included 10 patients; all patients had a fasting period of 8 hours before the operation to avoid aspiration of the gastric contents during the operative procedures. The operation was performed under general anesthesia through nasotracheal intubation. After that $1.8 \mathrm{ml}$ of local anesthetic agent (mepecaine-L,1 120000 levonordefrin) for hemostasis intraorally around the planed area was injected intraorally as field Block.

2. In the mandible, the surgical procedure was standardized for all patients. para-crestal incision was made buccally just parallel to the alveolar ridge of the mandible extending from retromolar area to the area of lower second premolar (Figure:1a).

3. Muco-periosteal flap was reflected using sharp muco-periosteal elevator exposing the edentulous posterior atrophic mandibular ridge (Figure: 1b).

4. After carefully raising the flap (gauze impregnated with $0.9 \%$ saline) was packed in the surgical site intra-orally.

5. Anterior superior iliac spine was identified as a land mark and the iliac crest medially \&laterally was grasped to identify contour of the iliac crest.

6. Skin incision was done starting $1 \mathrm{~cm}$ away from ASIS extending 5-6 $\mathrm{cm}$ posteriorly.

7. Dissection through the subcutaneous fascia fats on lateral aspect of the crest was done.

8. Incision using 10\# blade on the sling between tensor fascia lata muscle laterally \& external abdominal oblique muscle attachment medially was done.

9. Subperiosteal dissection on medial aspect of iliac bone was done. 
10. Retraction of abdominal muscles medially \& exposing the medial surface.

11. The needed graft was harvested according to the defect size by trephine bur, and then bi beveled chisel and mallet were used by gentle tapping until separation of the cortico-cancellous bone graft with taking care.

12. The graft was placed in saline until checking the donor site thoroughly to make sure there is no perforation in the lateral cortex and to check whether there is abnormal bleeding or not (abnormal bleeding treated by local homeostatic measures.

13. The cortico-cancellous graft block was prepared by milling it by using the bone milling machine to get cortico-cancellous particulate bone graft then packing the particulate in a plastic syringe and Frios Algipore was mixed with the autogenous bone graft to be ready to be packed in recipient site.

14. The recipient site (posterior mandible) was prepared for receiving the graft by making fenestrations using a round bur.

15. The titanium mesh was fixed to the buccal and lingual bony plates by bone tacks ( 2 screws on buccal side and 2 on the lingual side) (Figure: 1c).

\section{Postoperative evaluation:}

\section{1- Clinical evaluation:}

A- Intraoral evaluation: The patients were clinically examined post operatively 1 st day, $1^{\text {st }}$ week, $2^{\text {nd }}$ week, $1^{\text {st }}$ month, $2^{\text {nd }}$ month to evaluate the integrity of the wound mucoperiosteal flap during healing period.

B- Extra oral evaluation of the patients at the iliac wound to determine the absence of any signs of infection or inflammation (redness, hotness, swelling of skin), Furthermore, Absence of any gait disturbance. After clinical evaluation of the wound, the iliac sutures were removed 1 week postoperatively.

\section{2- Radiographic evaluation:}

- CBCT was used to evaluate vertical height and width after augmentation in both one week and after 6 months.

- Linear measurements obtained at three points $(\mathrm{A}, \mathrm{B}, \mathrm{C})$ in each augmented site from inferior border of the mandible to the crest of the augmented ridge 1 week postoperatively and after 6 months then calculate the mean height.

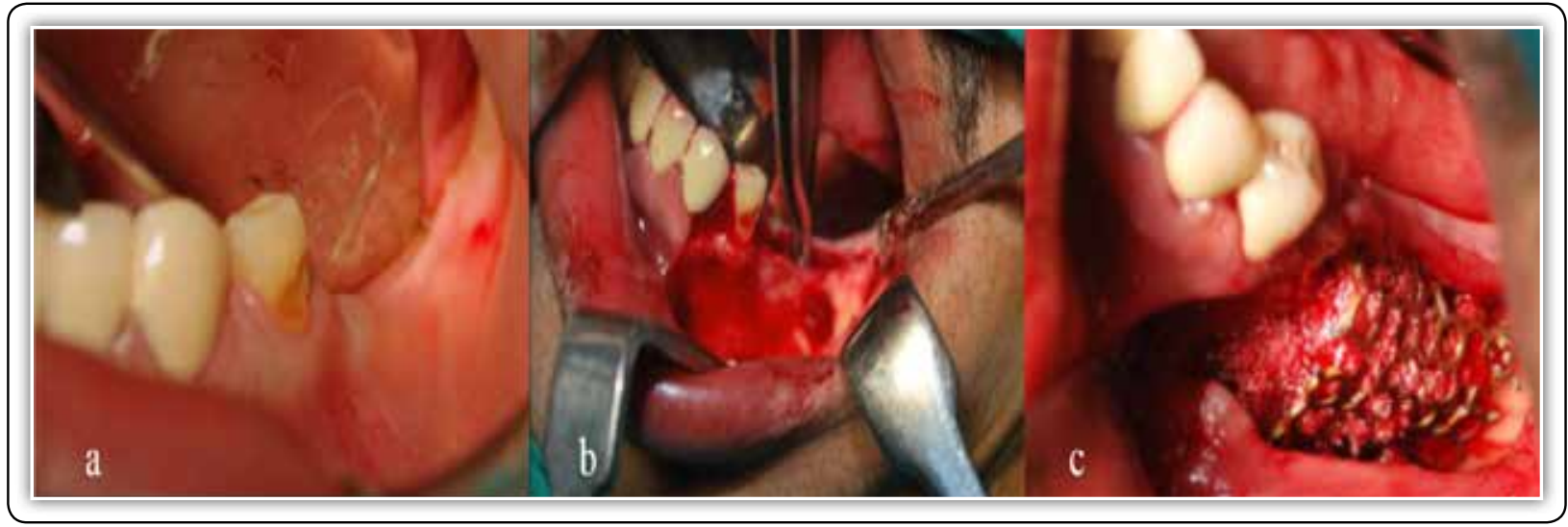

Fig. (1) a: The alveolar ridge atrophy in the mandible before the procedure; b: Reflection of the muco- periosteal flap; and c: Packed autogenous bone graft mixed with Algipore under titanium mesh. 


\section{Second surgery:}

After 6 months postoperatively the titanium mesh was removed by unscrewing the titanium screws before implant installation.

\section{RESULTS}

\section{Evaluation of the augmented Bone:}

The augmented alveolar ridge was evaluated digitally by CBCT scans. The augmented ridge was reconstructed by autogenous bone which was harvested from the iliac crest bone marrow and mixed with Frios Algipore. The mesh was removed about 6 months after insertion. The vertical bone height and width was accepted for successful dental implant placement in eight out of ten patients, others showed deficient vertical dimension during mesh removal. Dental implants were placed guided by a surgical template that were prefabricated that required re-grafting and delayed implant placement.

\section{Radiographic results:}

a- Vertical bone height: The difference between the preoperative, immediate, three months and six months postoperative was statistically significant $(\mathrm{P}$ value $=0.001)$ (Table1).

b- Horizontal Bone width (Buccolingually): The difference between the preoperative, immediate and six months postoperative was statistically significant $(\mathrm{P}$ value $=0.001)$. (Table 1).

\section{Post-surgical complications:}

Several complications were observed early after the augmentation procedure and implant placement such as post-operative pain, hematoma and swelling. These complications disappeared after 4-5 postoperative days. Mesh exposure was observed in six cases out of ten cases $(n=60 \%)$. In these cases which showed mesh exposure, weekly to these cases were carried out. Patients were instructed to apply saline twice daily.

The exposed titanium mesh was removed at the same time of implant placement. Temporary paresthesia of the lower lip was observed in two cases of all (n 20\%) with no incidence of anesthesia or dysesthesia. In all cases it disappeared spontaneously after few weeks. Mild edema was observed after the augmentation procedure intraorally which resolved in 5-7 days postoperatively in eight cases of the study. Hematoma was observed in four cases. Implants was placed successfully in eight patients out of ten, four immediately after the operation of the removal of the Ti mesh after 6 months. Postoperatively, four patients needed more graft and more time to wait, and two failed due to early mesh exposure and loss of most of the graft.

The quantitative evaluation of augmented bone measured by CBCT after six months postoperatively: Mean Horizontal width gain by CBCT was $3.4 \pm 2 \mathrm{~mm}$ and Mean vertical bone gain by CBCT was $5.4 \pm 3.4 \mathrm{~mm}$.

TABLE (1): Preoperative, immediate ,3 months and 6 months postoperative bone height and bone width.

\begin{tabular}{|c|c|c|c|c|c|}
\hline & Minimum & Maximum & Mean & SD & $P$ value \\
\hline \multicolumn{6}{|l|}{ Bone height } \\
\hline Preoperative & $6.2 \mathrm{~mm}$ & $8.4 \mathrm{~mm}$ & $7.2 \mathrm{~mm}$ & & \multirow{4}{*}{$0.001^{*}$} \\
\hline Immediate & $10.6 \mathrm{~mm}$ & $14.6 \mathrm{~mm}$ & $15 \mathrm{~mm}$ & 1.26 & \\
\hline 3 months postop & $8 \mathrm{~mm}$ & $13.8 \mathrm{~mm}$ & $13.5 \mathrm{~mm}$ & 2.075 & \\
\hline 6 months postop & $8 \mathrm{~mm}$ & $13.3 \mathrm{~mm}$ & $11.5 \mathrm{~mm}$ & 2.11 & \\
\hline \multicolumn{6}{|l|}{ Bone width (buccolingually) } \\
\hline preoperative & $3 \mathrm{~mm}$ & $4.5 \mathrm{~mm}$ & $3.7 \mathrm{~mm}$ & & \multirow{4}{*}{$0.001^{*}$} \\
\hline Immediate & $6.5 \mathrm{~mm}$ & $8.8 \mathrm{~mm}$ & $7.5 \mathrm{~mm}$ & 0.691 & \\
\hline 3 months postop. & $4 \mathrm{~mm}$ & $8.6 \mathrm{~mm}$ & $6.9 \mathrm{~mm}$ & 1.496 & \\
\hline 6 months postop & $3 \mathrm{~mm}$ & $8.6 \mathrm{~mm}$ & $6.8 \mathrm{~mm}$ & 1.74 & \\
\hline
\end{tabular}




\section{DISCUSSION}

The purpose of this study was to evaluate the vertical and horizontal ridge augmentation using: titanium mesh in atrophic mandible. To do this, results from linear measurements of vertical height and horizontal width of the ridge after augmentation with autogenous iliac graft mixed with algipore. These measurements were obtained from segmented planrneca romexis CBCT scans. In a systemic review, utilization of a TiMe as a barrier resulted in $4.91 \mathrm{~mm}$ of vertical ridge augmentation and 4.36 $\mathrm{mm}$ of horizontal augmentation. It should be noted that comparing various studies should be performed with caution because different graft materials and different techniques have been implemented in the published literature. However, despite the divergence of grafting materials and techniques ${ }^{(2)}$.

There is a controversy in the literature regarding the effect of TiMe on bone volume. While several authors have reported no effect of the exposure on the volume of bone grafting. Others have reported that bone loss occurs when the TiMe is exposed. Despite the reported loss of bone volume, when this occurred, the obtained bone volume was sufficient to place implants. However, von Arx at el ${ }^{17)}$ experienced a clinical situation in which the exposure of the TiMe resulted in significant bone loss that precluded placement of implants. The effect of the size of the exposure, the timing of the exposure after the initial ridge augmentation procedure, and the type of graft material on the final clinical outcome are elements that need to be studied.

There is a scarcity in the literature regarding the quality of the obtained osseous tissue when exposure of the TiMe occurs. Proussaefs et al ${ }^{(18)}$ published a case series report in which histologic specimens were obtained and analyzed from alveolar ridges where a localized alveolar ridge augmentation procedure was performed by using a TiMe as a barrier. In their study, in which histomorphometric analysis was performed, the exposed sites had lesser bone formation while a bigger portion of connective tissue was present at the augmented sites associated with mesh exposure. It might be suggested that in addition to the reduced bone volume, exposure of the mesh may result in decreased quality of the augmented alveolus.

Boyne et al ${ }^{(14)}$ observed the presence of a newly formed connective layer along with granulation tissue under the mesh ("pseudo periosteum"). The presence of abundant granulation tissue underneath the mesh may indicate placement of implants at a later stage. The clinical significance of this connective and granulation tissue layer is unknown. Proussaefs et al ${ }^{(18)}$ suggested that the micromovement of the titanium barrier could induce the formation of this layer of connective and granulation tissue. While this suggestion has not been validated, removing the portion of the exposed mesh may result in lesser TiMe micromovement and lesser amount of granulation tissue formation ${ }^{(19)}$.

Non-resorbable membrane barriers such as titanium mesh when they exposed in the oral cavity after GBR, infection may occur and that can jeopardize the results on the contrary, exposure of the Ti-mesh did not appear to affect the final outcome of the vertically augmented ridge. This is in accordance with von Arx et al, ${ }^{(17)}$ Roccuzzo et al. (2004) ${ }^{(12)}$. The mesh exposure not seemed to be the cause of severe bone resorption, and it could tolerate infection. In the case of mesh removal due to the severe infection partial bone resorption would occur and total bone resorption has not been observed". In our study, the Ti-mesh was exposed in six patients out of ten patients which is better than Her S. et al ${ }^{(20)}$ who reported exposure of titanium mesh in 7 of the 27 surgical sites evaluated. They finally concluded that substantial bone augmentation can be achieved using titanium mesh in conjunction with bone grafting. Furthermore, exposure of 
titanium mesh during healing period does not necessarily compromise the final treatment outcome The Ti-mesh was removed at the time of implant placement in all cases ${ }^{(21,22)}$. The presence of a thin granulation tissue around the titanium mesh did not simplify its removal in a separate approach as said by Proussaefs et al. ${ }^{(18)}$.

In our study the mean time of healing after GBR was 6 months after the first surgery. This period of time is less than that reported by other authors in their previous study. After 6 months of bone grafting, healing of grafted bone occurred which allowed loading of the implants in this graft in all cases of the study. Longer time was needed before implant placement to ensure healing of the grafted bone as presented in previous studies ${ }^{(23)}$.

\section{CONCLUSION}

Within the limitations of this in vivo study, it was concluded that excellent bone augmentation results both vertically and horizontally can be achieved, except for those of titanium mesh exposure.

\section{REFERENCES}

1. Jemt T, Lekholm U. Single implants and buccal bone grafts in the anterior maxilla: measurements of buccal crestal contours in a 6-year prospective clinical study. Clin Implant Dent Relat Res 2005; 7:127-135.

2. Gaggl AJ, Bürger HK, Chiari FM. Free microvascular transfer of segmental corticocancellous femur for reconstruction of the alveolar ridge. Br J Oral Maxillo fac Surg 2008; 46:211-217.

3. Chiapasco M, Romeo E, Casentini P, Rimondini L. Alveolar distraction osteogenesis vs. vertical guided bone regeneration for the correction of vertically deficient edentulous ridges: a 1-3-year prospective study on humans. Clin Oral Implants Res 2004; 15:82-95.

4. Takahashi T, Inai T, Kochi S. Long-term follow-up of dental implants placed in a grafted alveolar cleft: evaluation of alveolar bone height. Oral Surg Oral MedOral Pathol Oral Radiol Endod 2008; 105:297-302.
5. Merli M, Migani M, EspositoM. Vertical ridge augmentation with autogenous bone grafts: resorbable barriers supported by osteosynthesis plates versus titanium-reinforced barriers: a preliminary report of a blinded, randomized controlled clinical trial. Int J Oral Maxillo fac Implants 2007; 22:373-382.

6. Engelke WG, Diederichs CG, Jacobs HG. Alveolar reconstruction with splitting osteotomy and micro fixation of implants. Int J Oral Maxillo fac Implants 1997; 12:310 316.

7. Jensen OT, Cockrell R, Kuhike L, Reed C. Anterior maxillary alveolar distraction osteogenesis: a prospective 5-yearclinical study. Int J Oral Maxillo fac Implants 2002; $17: 52-68$.

8. Uckan S, Dolanmaz D, Kalayci A. Distraction osteogenesis of basal mandibular bone for reconstruction of the alveolar ridge. Br J Oral Maxillo fac Surg 2002; 40:393-39.

9. Buser D, Dula K, Hirt HP. Lateral ridge augmentation using autografts and barrier membranes: a clinical study with 40partially edentulous patients. J Oral Maxillo fac Surg 1996; 54:203-209.

10. Schopper C, Goriwoda W, Moser D. Long term results after guided bone regeneration with resorbable and microporous titanium membranes. Oral Maxillo fac Surg Clin N Am 2001; 13:449-455.

11. Donos N, Kostodopulos I, Karring T. Alveolar ridge augmentation using a resorbable copolymer membrane and autogenous bone grafts: an experimental study in the rat Clin Oral Implants Res 2002; 13:449-456.

12. Roccuzzo M, Ramieri G, Spada MC, Bianchi SD, Berrone $S$. Vertical alveolar ridge augmentation by means of a titaniummesh and autogenous bone grafts. Clin Oral Implants Res 2004; 15:73-81.

13. Roccuzzo M, Ramieri G, Bunino M, Berrone S. Autogenous bone graft alone or associated with titanium mesh for vertical alveolar ridge augmentation: a controlled clinical trial. Clin Oral Implants Res 2007; 18:286-294.

14. Boyne PJ. Reconstruction of the maxilla and mandible. Quintessence Inc, Hanover Park 1997; pp 37-52.

15. Louis PJ, Gutta R, Said-Al-Naief, Bartolucci . Reconstruction of the maxilla and mandible with particulate bone graft and titanium mesh for implant placement. J Oral Maxillofac Surg 2008; 66:235-245. 
16. Weng D, Hürzeler MB, Quiones CR. Contribution of the periosteum to bone formation in guided bone regeneration. Clin Oral Implants Res 2000; 11:546.

17. Von Arx T., Hardt N. and Wallkamm B. The TIME technique: a new method for localized alveolar ridge augmentation prior to placement of dental implants. Int. J Oral Maxillofac. Implants. 1996; 11: 387-94.19-Albrektsson T., Branemark P.I., Hansson H. A. and Lindstrom J. Osseointegrated titanium implants. Requirements for ensuring along-lasting, direct bone to-implant anchorage in man. Acta Orthop. Scand 1981; 52: 155-70.

18. Proussaefs P., Lozada J., Kleinman A., Rohrer M.D. and McMillan P. J. The use of titanium mesh in conjunction with autogenous bone graft and inorganic bovine bone mineral (bio-oss) for localized alveolar ridge augmentation: a human study. Int. J Periodontics Restorative Dent. 2003; 23: 185-95.

19. Antoun H., Sitbon JM., Martinez H. and Missika PA. prospective randomized study comparing two techniques of bone augmentation: onlay graft alone or associated with a membrane. Clin. Oral Implants Res. 2001; 12: 632-9.

20. Her, S., Kang, T., \& Fien, M. J. Titanium Mesh as an Alternative to a Membrane for Ridge Augmentation. J Oral Maxillofac Surg 2012, 70(4), 803-810.

21. Stringer D, Boyne P: Modification of the maxillary step osteotomy and stabilization with titanium mesh. J Oral Maxillofac Surg.1986; 44:487.

22. Miyamoto I., Funaki K. Yamauchi K., Kodama T. and Takahashi T. Alveolar ridge reconstruction with titanium mesh and autogenous particulate bone graft: computed tomography-based evaluations of augmented bone quality and quantity. Clin. Implant Dent. Relat. Res. 2012; 14:304-11.

23. Corinaldesi G, Pieri F, Marchetti C, Fini M, Aldini NN, Giardino R. Histologic and histomorphometric evaluation of alveolar ridge augmentation using bone grafts and titanium micromesh in humans. J. Periodontal.2007; 78: 1477-84. 